\title{
Perspectives of Overdamped Josephson Junctions in Voltage Standard Applications
}

\author{
Vincenzo Lacquaniti, Natascia De Leo, Matteo Fretto and Andrea Sosso*
}

Istituto Nazionale di Ricerca Metrologica, Strada delle Cacce 91, 10135 Torino, Italy

\begin{abstract}
We discuss the main characteristics that must be satisfied by Josephson junctions for metrological applications and, specifically, in the development of voltage standards of new generation. Data reported in the literature for the most established technologies, like SNS (Superconductor-Normal conductor-Superconductor), SINIS (SuperconductorInsulator-Normal conductor-Insulator-Superconductor) and others, are analyzed and compared. The properties of $\mathrm{Nb} / \mathrm{Al}$ $\mathrm{AlO}_{\mathrm{x}} / \mathrm{Nb}$ overdamped SNIS (Superconductor-Normal conductor-Insulator-Superconductor) Josephson junctions developed at INRiM are also presented, since these junctions can optimize crucial parameters such as the current density and the characteristic voltage. Operation at temperatures above $4.2 \mathrm{~K}$ is also discussed, in view of using cryocooler setups to replace liquid helium refrigeration systems.
\end{abstract}

\section{INTRODUCTION}

Josephson junctions used nowadays in DC voltage standard applications are based on hysteretic SIS (Superconductor-Insulator-Superconductor) junctions with zero crossing steps, i.e. voltage steps whose current range spans positive and negative values, including the condition of zero DC bias. The choice of this technique dates back to the first attempts in series-connecting the thousands of junctions needed to reach output levels of $1 \mathrm{~V}$ and above, as required in metrological applications. Exploitation of the zero crossing steps, fist suggested by Levinsen [1], eventually allowed to overcome many technological difficulties and made it possible to realize arrays with reproducible overlapping steps, providing DC voltages up to $10 \mathrm{~V}$. The availability of $10 \mathrm{~V}$ standards with quantum accuracy has led to dramatic improvements in DC voltage metrology, and it is now possible in primary DC voltage calibrations at $10 \mathrm{~V}$ to attain relative uncertainties as low as $10^{-11}$.

More recently, the interest in voltage standard research has moved to the investigation of techniques for extending the application of Josephson arrays to AC quantum standards and to standards for arbitrary time-varying signals. To this aim, junctions with non hysteretic behavior were suggested to allow changing the output voltage through control of the bias current. The substantial difference, from the application viewpoint, in using non hysteretic junctions, is that their IV curve (voltage $v s$. current relationship) under irradiation is a one to one staircase, thus the output voltage is univocally defined by the current feed through the bias circuit. This is not the case for hysteretic junctions used in DC standards, were steps are overlapping and all share approximately the same interval of currents.

In the so-called programmable standards, the junctions bias currents are used to activate/deactivate array sections. Such arrays are typically subdivided in sub-circuits with

*Address correspondence to this author at the Istituto Nazionale di Ricerca Metrologica, Strada delle Cacce 91, 10135 Torino, Italy;

E-mail: a.sosso@inrim.it series connected junctions generating voltages following a power of two sequence (see Fig. 1). Combining the sections it is then possible to source binary programmed voltages in a way that is very similar to the technique used in electronic digital to analog converters [2]. In order to replace best AC standards, the uttermost accuracy has to be reached and many efforts have been devoted in realizing arrays with performances suited to the tight requirements set by modern primary metrology. Many approaches to junction fabrication have been developed, and several different technologies have proven successful in providing voltages up to $10 \mathrm{~V}$, with good metrological properties. Programmable Josephson arrays are so far the most successful attempt to extend metrological applications of Josephson standards beyond dc. Programmable arrays operating at $1 \mathrm{~V}$ have been effectively used for several applications: as traveling standards for international comparisons [3], for generating precisely varying voltages in a watt balance [4] as quantum impedance and power standards [5]. Moreover, only programmable standards can presently provide output voltages up and above $1 \mathrm{~V}$, and even exceeding $10 \mathrm{~V}$ [6].

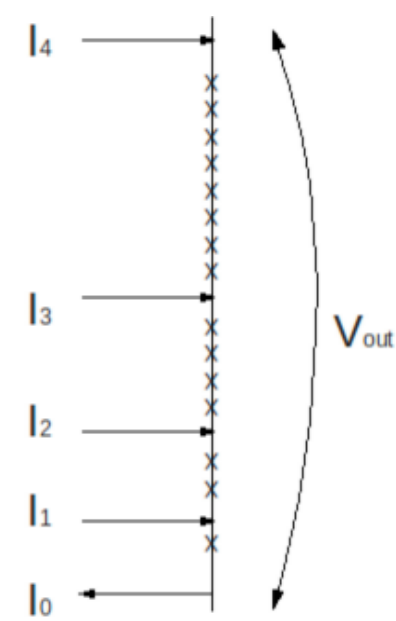

Fig. (1). Operating principle of programmable Josephson arrays. By controlling the bias currents $\mathrm{I}_{1}-\mathrm{I}_{4}$ it is possible to change the output voltage $\mathrm{V}_{\text {out }}$.

2010 Bentham Open 
Nonetheless, this technique suffer from some limitations, the most severe is due to the time for step switching, where junctions are not operating in a quantized state. During these transients, the array voltage is not precisely known and the uncertainty of the generated signal increases with the fraction of period spent in the transients. Since the minimum transient time is constrained by technological limitations, programmable arrays can fulfill primary metrology uncertainties only for signals with frequencies up to few hundreds $\mathrm{Hz}$ [7].

To overcome limitations of programmable standards, arrays operating with a pulsed, square wave, rf signal have been developed. Using short pulses instead of a sinusoidal rf signal makes it possible to effectively modulate the signal period while keeping junctions phase locked over a wide range of frequencies [8-10]. Fundamental accuracy follows from the control of the flux quanta transferred through the junctions by the pulsed signal. The output voltage is then exactly calculable in terms of fundamental constants if the number of the flux quanta per unit time, i.e. the pulse repetition rate is known [11]. Pulsed standards allow to synthesize arbitrary waveforms with quantum accuracy based on the sigma-delta technique for digital to analog conversion developed for semiconductor electronics and very high spectral purity [12]. Both operation and fabrication of pulsed standards set very challenging problems. Due to the complexity of pulse waveform, the apparatuses for generation of precisely frequency controlled pulses are sophisticated and expensive, and the design of $\mathrm{rf}$ transmission lines is extremely difficult because of the harmonic richness of the signal. In addition, it is extremely difficult to generate a bipolar output with a frequency modulated Josephson array, and very complex AC biasing techniques, involving a sine wave and a pulsed signal, both synchronized, must be used for real AC operation [12]. Generation of the pulse train for proper junction operation requires top-end instrumentation and unavoidably limits the signal fundamental frequency to values much lower than those obtainable with continuous wave sources. Power distribution to array junctions is also of concern, since the usual microwave techniques developed for nearly monochromatic signals are not directly applicable to broadband pulses. Adoption of lumped circuit methodologies seems at present the most viable solution, though highly demanding on the fabrication side. In order to have negligible effects on circuit behavior, the propagation time of rf signal along the array must be smaller than the signal period, i.e. the array dimensions must be smaller than the signal wavelength $\lambda$. To guarantee reliable operation, $\lambda / 8$ is typically considered the maximum value acceptable for array dimensions [13]. Despite all these difficulties, arrays with as many as 10000 junctions have been successfully fabricated, providing synthesized voltage signal exceeding $200 \mathrm{mV}$, with quantum accuracy and extreme spectral purity [13].

A new technique has been recently proposed to overcome some of the difficulties encountered with programmable and pulsed standards. The method, named Pulse Power Modulation (PPM), is based on a controlled activation/deactivation of the driving rf signal, to simultaneously set every junction in the array into either one of two states: zero voltage in absence of rf signal and a quantum defined step voltage upon rf application. Waveform synthesis can then be realized by
Pulse Width Modulation of the array voltage [14]. The requirements in junction technology for PPM are different form those set by programmable and pulsed standards, since for proper operation an IV curve where the interval of currents of the relevant steps partially overlaps the critical current is needed. Such a behavior can be obtained if a precise control over junction hysteresis is feasible in the fabrication stage, to provide an intermediate degree of hysteresis, between those of the fully hysteretic SIS for DC and the non hysteretic SNS.

Although limited in space, this overview wouldn't be complete without mentioning RSFQ (Rapid Single Flux Quantum) as an alternative technique to synthesize arbitrary and AC signals with quantum voltage accuracy. RSFQ has been, and still remains, a very active field of research for quantum digital electronics applications, yet developments for voltage standards have always been left out of the mainstream of research interests in Metrology [15]. One of the main reasons for that is most likely to be found in the completely different approach, know-how and experiences involved by RSFQ, with respect to the common background of voltage metrologist. In RSFQ standards, accurate voltage signals are generated by controlling flux quantized by a Josephson junction. Flux quanta generation is "triggered" by a pulse sequence, thus can be precisely timed, in analogy with the "phase lock" process exploited in array standards [16]. RSFQ approach is potentially advantageous in that the complex and expensive microwave apparatus needed for ordinary standards is avoided, the drive signal being generated by the superconductive circuit itself. The only requirement for RSFQ circuits is an external accurate frequency reference, typically operating in the $\mathrm{MHz}$ range. The simplification in instrumentation must be traded off with a much higher complexity of superconductive circuit, namely an increased number of junctions, additional elements like inductors, and fabrication of junctions with different parameters in the same device. The basic element in RSFQ is the Josephson transmission line, a string of Josephson junctions connected by inductors where the pulse can propagate, like in transmission lines, and even amplified [17]. A basic Digital to Analog converter suitable for voltage standard will include at least some voltage multiplier stages, to increase the output signal to practical values (See Fig. 2). Coupling between Josephson transmission lines and voltage multipliers is obtained by capacitive coupling [18], but magnetic coupling through transformer-like circuits has proven to be more effective [19]. Nowadays DAC for Metrology are fairly more complex devices, with many digital blocks performing various specialized functions, and correspondingly high power consumption. Successful operation, of a 10-bit RSFQ DAC capable of generating up to $20 \mathrm{mV}$ has recently been reported [20].

\section{CURRENT JUNCTION TECHNOLOGIES AND FABRICATION ISSUES}

The fabrication of arrays for AC generation and waveform synthesis is a difficult, challenging task, and many different technologies have been proposed and tested, yet the choice of material and fabrication techniques still represents an open question. Independent of the technique adopted for signal generation, a relevant problem is posed by the high 


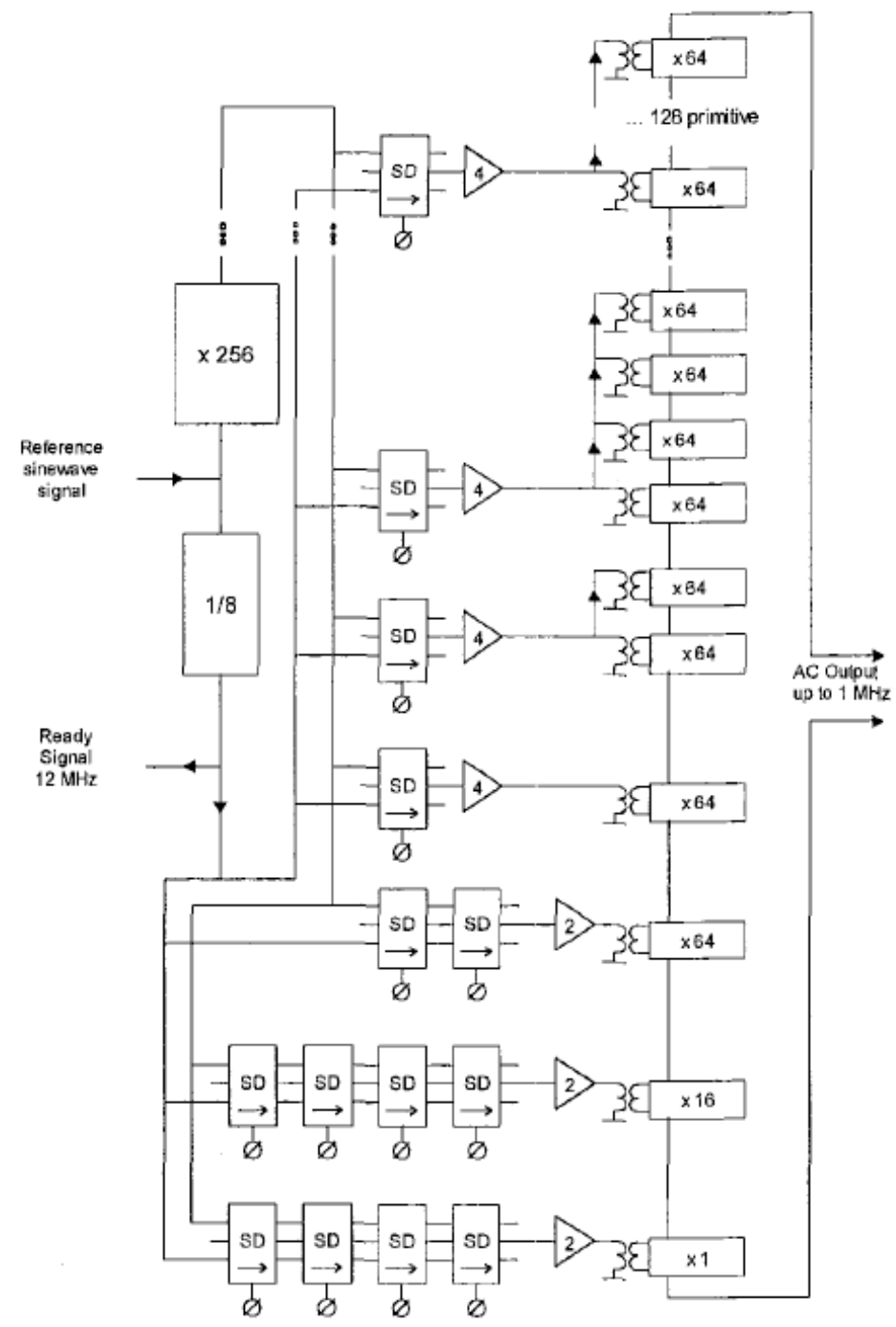

Fig. (2). Simplified schematic of a Digital to Analog converter realized with RSFQ technology (from [16]).

number of junctions needed to reach practical voltages, since junctions for AC typically operate on the first step and the drive frequency is limited for technical and economical reasons [21]. A high noise immunity, low power dissipation, reduced dimensions are also essential properties in metrological applications and in view of spreading quantum standards to a wider range of users.

The chip dimensions are set by the area and the number of junctions, both essential parameters for technology, because a reduced area along with a high number of junctions make it difficult to guarantee the uniformity of their electrical properties, which is essential to observe decent collective steps.

These requirements can be translated into well-defined specifications on junction parameters. First, the critical current $I_{c}$, that sets an upper limit on the amplitude of the quantized steps, should be large enough for steps with suitable width and noise immunity, yet a too high value increases dissipation in junctions. The area of the junction must be small, to reduce array dimensions, but avoiding the excessive difficulties in fabrication imposed by deep submi- cron patterning, today still hard to achieve for high integration superconductive circuits. It follows that an optimal range exist for critical current density. Presently, values ranging from 10 to $100 \mathrm{kA} / \mathrm{cm}^{2}$ appear to be the best choice.

The characteristic voltage $V_{c}=I_{c} R_{N}$ with $R_{N}$ junction normal tunneling resistance, determines the microwave optimal drive frequency $f_{d}$, from the relation $\Omega=f_{d} / f_{c}$, where $f_{c}=(2 e / h) V_{c}$ and thus the step voltage (i.e. the voltage resolution of the array) and the number of junctions needed to achieve the maximum requested voltage output. To obtain the maximum step amplitude, $\Omega$ must be $\approx 1$ [22]. In order to use commercial microwave instrumentation and reduce as much as possible the number of junctions, drive frequencies close to $70 \mathrm{GHz}$ are used, and $V_{c}$ around $150 \mu \mathrm{V}$ are needed. Of course even larger values, which on the other hand are absolutely advantageous for speed applications, can be used. But in voltage standard application this causes a sensible reduction of the step width.

The characteristic voltage defines also the highest speed of RSFQ circuits since this is proportional to $\tau_{c}=1 / f_{c}$, In this case, the highest $V_{c}$ and the higher the speed. 
A challenging problem to be solved in the next future for voltage metrology and superconductive electronics applications, is operation in cryocoolers at a temperature greater than $4.2 \mathrm{~K}$. Indeed, in order to make the Josephson quantum standards available to a widespread market, rather than limited only to the National Laboratories, as well as affordable for private companies needing an accurate voltage reference, refrigeration systems cheaper and more compact than those nowadays used for niobium junctions are required.

At present, however, large arrays fabricated with higher critical temperature superconductors like YBCO (Yttrium Barium Copper Oxide), or the more recent $\mathrm{MgB}_{2}$ are not yet available, since the technology of these junctions do not allow to achieve the required integration level $[23,24]$. The best results have been achieved by shunted bicrystal YBCO junctions, where voltages near to $30 \mathrm{mV}$ have been measured at $77 \mathrm{~K}$ [23].

Therefore in this paper we discuss extensively niobium and niobium nitride based junctions, considering high $T_{c}$ junctions in the section devoted to the use at temperatures above $4.2 \mathrm{~K}$.

Considering the electrode configuration, non-hysteretic IV characteristic can be obtained by three main classes of junctions:

- hysteretic SIS with external shunt

- metallic barrier SNS

\section{- double barrier SINIS}

The first class, directly derived from the most developed and optimized process of superconductive electronics, and which is still the predominant technology for RSFQ circuits, suffers from the disadvantage of a configuration requiring an external resistor or a more complex circuitry. This, with the severe limitation to $I c$ from chaotic instabilities [25] limits the use of these junctions in voltage standard circuits. Therefore we will not consider junctions of this type in the following, where a detailed analysis limited to the last two classes will be carried out.

\subsection{Programmable Voltage Standards}

Programmable voltage standards (PJAVS) are made with series-connected subarrays, whose size follows a power of two sequence. Through control of the bias current of each section, the series voltage is set by the code represented by on/off status of array bias lines. To switch subarrays, junctions that can generate a current-controlled, univocally defined voltage, i.e. junctions with a single valued (nonhysteretic) IV curve must be used. To provide signal to noise ratios adequate to metrological applications, critical currents at the $\mathrm{mA}$ level are at least required. On the other side, due to the fact that only the first Shapiro step of junctions is used, to achieve voltages of 1 and $10 \mathrm{~V}, V_{c}$ even in excess of 100 $\mu \mathrm{V}$ are sought to reduce number of junctions.

As known, the amplitude of the $\mathrm{n}=1$ quantized Shapiro step normalized to the critical current attains its maximum when the microwave drive frequency and the junction characteristic frequency are nearly equal. This corresponds also to a condition of minimal power dissipation [22]. This leads to optimal $V_{c}$ of $100-200 \mu \mathrm{V}$.
SINIS junctions achieve the shunting of the capacitance of a SIS junction, by using two, very thin oxide layers, separated by a metallic barrier. They are typically made of $\mathrm{Nb} /$ $\mathrm{Al} / \mathrm{AlO}_{\mathrm{x}} / \mathrm{Al} / \mathrm{AlO}_{\mathrm{x}} / \mathrm{Al} / \mathrm{Nb}$ and have been extensively studied both theoretically and experimentally [26-30]. The best results on these junctions for superconductive electronics applications and especially for programmable voltage standard have been obtained by the PTB group, who realized complete successful circuits for programmable potentiometer at 1 and $10 \mathrm{~V}$ level with 7000 and 70.000 junctions respectively. These SINIS junctions feature $J_{c} \leq 1 \mathrm{kA} / \mathrm{cm}^{2}, V_{c}$ as high as more than $250 \mu \mathrm{V}$ at $4.2 \mathrm{~K}$ with junction size 100 $1000 \mu \mathrm{m}^{2}$ [31]. SINIS junctions with various values of $\mathrm{Al}$ thickness and $\mathrm{AlO}_{\mathrm{x}}$ barrier transparency have been reported, but, even if feature $J_{c}$ and $V_{c}$ higher than those given above, their IV characteristic was rather anomalous showing an high residual hysteresis at $4.2 \mathrm{~K}$, and they were hardly reproducible [32, 33].

In general a problem for the SINIS junctions is due to the high transparency of the two insulating barriers, with the necessity to realize very smooth planarization of the underlying films, since the probability of defects such as pinholes is increased. The fabrication process, requiring two barriers and three thin aluminum layers highly homogenous, has limitations for the fabrication of really high number of junctions, as in arrays for programmable voltages operating at $10 \mathrm{~V}$ and more [34].

In SNS junctions the damping of the IV characteristic for non-hysteretic behavior is obtained by using a normal metal as barrier. SNS have high values of $J_{c}$, but typically the metals used have very low resistivities. $\mathrm{Nb} / \mathrm{PdAu} / \mathrm{Nb}$ junctions have been the first developed type of SNS junctions for this application by NIST group, and have produced stable voltage outputs at $1 \mathrm{~V}$ as binary arrays for programmable potentiometers, being also used in circuits for pulse driven $\mathrm{AC}$ synthesis $[11,12]$. They feature values in the range of $100 \mathrm{kA} / \mathrm{cm}^{2}$ and $V_{c}$ between 5 and $30 \mu \mathrm{V}$ with typical size of few $\mu \mathrm{m}^{2}$, even if $J_{c}$ values would support also submicron dimensions. The values of $V_{c}$ limit their use to drive frequencies of few $\mathrm{GHz}$.

Impressive results have been obtained with $\mathrm{NbN} / \mathrm{TiN} /$ $\mathrm{NbN}$ junctions, developed by the Tsukuba group, since very large arrays, with more than 300.000 series connected junctions have been successfully tested in a 8 and 11 bit DAC circuits with quantized steps at $10 \mathrm{~V}$ at $10 \mathrm{~K}[35,36]$. Features of these junctions are $J_{c}$ about $10^{4} \mathrm{~A} / \mathrm{cm}^{2}$ and $V_{c} 10$ $20 \mu \mathrm{V}$ at $10 \mathrm{~K}$, with areas of few square micrometer.

Materials have been also proposed as normal metal barrier which are at the metallic insulator transition. In such a way it was possible to tune the barrier resistivity and the characteristic voltage of the junctions [37, 38]. Among the experimental results we mention $\mathrm{Nb} / \mathrm{TaO}_{\mathrm{x}} / \mathrm{Nb}, \mathrm{NbN} / \mathrm{TaN} /$ $\mathrm{NbN}$ and $\mathrm{Nb} / \mathrm{Nb}_{\mathrm{x}} \mathrm{Si} / \mathrm{Nb}$ junctions. $\mathrm{Nb} / \mathrm{TaO}_{\mathrm{x}} / \mathrm{Nb}$ junctions fabricated at IEN-INRiM, showed resistivities varying different order of magnitudes depending on the bias voltage of the cathode during the sputtering deposition of the $\mathrm{TaO}_{x}$ film [39]. The junctions have however a marked aging, featuring a reduction of at values less than $10 \mathrm{~A} / \mathrm{cm}^{2}$. Also $\mathrm{NbN} / \mathrm{TaN} / \mathrm{NbN}$ junctions studied in [40] have not yet been suitable for large circuits production. These junctions featured very high $V_{c}$, up to more than $0.5 \mathrm{mV}$ at $4.2 \mathrm{~K}$ with 
$J_{c} 10^{4}-10^{5} \mathrm{~A} / \mathrm{cm}^{2}$, but required a difficult tuning of fabrication parameters and also the temperature dependence of these parameters was critical.

In this category, $\mathrm{Nb} / \mathrm{NbxSi} / \mathrm{Nb}$ junctions represent the most successful attempt sofar. Although previously studied by Barrera and Beasley in the ' 80 [41], the most promising results have been achieved recently by the NIST researchers, which experimented these junctions in multistacked arrays [42]. These authors achieved a transition from a conductive to an highly resistive phase by varying the sputter deposition power of the two elements. $J_{c}$ varying from 10 to $10 \mathrm{kA} / \mathrm{cm}^{2}$ and $\mathrm{V}_{\mathrm{c}}$ from few $\mu \mathrm{V}$ to $150 \mu \mathrm{V}$ and more at $4.2 \mathrm{~K}$ have been obtained. Outstanding results have been obtained within a cooperation between NIST and PTB [34], that jointly realized a programmable $10 \mathrm{~V}$ array with about 70,000 junctions.

The stabilization of the barrier stoichiometry, critical aspect of this type of junctions, can be achieved by a thermal annealing after the deposition of the trilayer [43].

\subsection{Pulsed Standards for Waveform Synthesis}

The series of pulses used for waveform synthesis has a very rich harmonic content and requires proper optimization of the microwave behavior in circuits for $\mathrm{rf}$ signal distribution. Most of the techniques adopted for analysis and design of circuits with distributed parameters assumes operation with narrow band signals, thus cannot be directly applied for pulse transmission. The most reliable solution to guarantee pulse operation is to reduce array dimensions allowing lumped analysis of circuit. To that aim array length must be shorter than $\lambda / 8$, where $\lambda$ is the wavelength of the microwave signal. This constraint sets strong requirements on junctions technology, in particular on junction size. In order to realize the array configuration described, materials resulting in improved steepness of the etch profile are preferred, especially when multi-stack junctions are employed.

SINIS junctions, initially developed for programmable voltage standards and previously described, have also been used for AC synthesis [28].

$\mathrm{Nb} / \mathrm{HfTi} / \mathrm{Nb}$ junctions have been experimented by PTB team with successful results in the realization of large submicrometric circuits for AC synthesis which have recently reached quantized voltages from 2000 series junctions [13]. These junctions feature about $80 \mathrm{kA} / \mathrm{cm}^{2}$ for HfTi $20 \mathrm{~nm}$ thick and of $15 \mu \mathrm{V}$ in $0.2 \mu \mathrm{m} \times 0.2 \mu \mathrm{m}$ junctions.

SNS junctions with similar low characteristic voltage, such as $\mathrm{Nb} / \mathrm{Ti} / \mathrm{Nb}$ junctions developed by Jena team and $\mathrm{Nb} / \mathrm{Al} / \mathrm{Nb}$ junctions studied at INRiM have not been resulted in large circuits yet $[39,40]$. Ti barrier junctions feature $j_{c}$ of $10^{4}-10^{5} \mathrm{~A} / \mathrm{cm}^{2}$ and $\mathrm{V}_{\mathrm{c}}$ from $10 \mu \mathrm{V}$ up to $100 \mu \mathrm{V}$, while for Al film $100 \mathrm{~nm}$ thick, also higher values of and similar or lower values for were obtained. In both case the junction size was in the micrometer and submicrometer range.

$\mathrm{Nb} / \mathrm{MoSi} / \mathrm{Nb}$ junctions also studied and experimented by NIST team, have been used to realize multistacked junctions for lumped arrays for AC synthesis [43, 44]. They feature critical current density between $10^{4}$ and $10^{5} \mathrm{~A} / \mathrm{cm}^{2}$, depending on barrier thickness, so that can be increased up to $200 \mu \mathrm{V}$ in junctions of few $\mu \mathrm{m}^{2}$.

\subsection{Power Control Standards}

Power control standards set some peculiar requisites on junction technologies, their operation being based on the overlapping of the range of currents of the steps and critical current. To fulfill these requirements, a tight control of junction hysteresis is needed.

Referring in particular to [45], where the junctions parameters for the overlapping of the $n=0$ and $n=1$ step are reported, it is pointed that the mentioned overlapping is obtained in a stable way when $f>(1.5-2) f_{c}$. In particular the authors suggest the adoption of edge type junctions with $V_{c}$ as high as $1 \mathrm{mV}$. However, in principle, no constraint on $V_{c}$ is needed, while the need of a sufficient, but not exceedingly high overlapping of the two steps is the only fundamental aspect.

First experimental results have been reported for SINIS junctions [46].

\subsection{Application to RSFQ}

The application of superconductive technology to RSFQ requires a high level of reliability, suitable for the development and testing of very complex circuits, with thousands of junctions implementing many different functional blocks. The best established technology, well mastered by many foundries, is based on SIS junctions, usually made of a $\mathrm{Nb} / \mathrm{AlO}_{\mathrm{x}} / \mathrm{Nb}$, with an externally shunt resistor [47]. The minimum junction area that can be fabricated with this technology is $12 \mathrm{um}$, and the whole process involves as many as 12 mask steps. Impressive results have been obtained with the standard $\mathrm{Nb} / \mathrm{AlO}_{\mathrm{x}} / \mathrm{Nb}$ technology: in [48] the operation of a RSFQ quantum DAC with 6000 Josephson junctions, subdivided in several functional blocks, is reported. SINIS junctions have proven to be suitable for RSFQ, and some fundamental circuits based on this technology were successfully operated [49]. More recently, owing to the wide range of tunability of their electrical parameters, co-sputtered Niobium-silicide barrier junctions appear interesting for a wide range of applications in digital electronics and well suited to RSFQ [50].

\section{NB/AL-ALO ${ }_{\mathrm{X}} / \mathrm{NB}$ OVERDAMPED SNIS JUNC- TIONS}

\subsection{Structure and General Properties}

Overdamped junctions developed at INRiM can be seen as an extended case of hysteretic $\mathrm{Nb} / \mathrm{Al}-\mathrm{AlO}_{\mathrm{x}} / \mathrm{Nb}$ SIS junctions, where the thickness of the Al, is increased up to 30-100 nm instead of the typical 5-10 nm, the oxidation dose being in the range 50-500 Pa.s. The niobium electrodes are $100-200 \mathrm{~nm}$ thick and the patterning of the base and top geometry is performed by lift-off and RIE respectively. Insulation via liquid anodization and deposition and patterning of the wiring layer are carried out accordingly to previously described recipes [51].

These junctions can be described as SNIS, since the thick aluminum film is a normal metal at liquid helium temperature. However, an essential feature is that, at $4.2 \mathrm{~K}$, a transition from the hysteretic to the non-hysteretic state can be induced, when the aluminum thickness is in the above 
mentioned range, by changing the $\mathrm{AlO}_{\mathrm{x}}$ exposure dose. Conversely, once chosen $\mathrm{Al}$ thickness and $\mathrm{AlO}_{\mathrm{x}}$ exposure values, this transition is observed as function of the junction temperature in measurements below $4.2 \mathrm{~K}$ [52].

A feature of overdamped SIS junctions of interest both in metrological applications and for high speed devices is the high value of current densities achievable, up to $10^{5} \mathrm{~A} / \mathrm{cm}^{2}$, along with $V_{c}$ up to several hundreds microvolt at $4.2 \mathrm{~K}$. Junctions with Al thickness ranging from 70-100 $\mathrm{nm}$ and exposure doses of $\mathrm{AlO}_{\mathrm{x}}$ between 150 and $250 \mathrm{~Pa} \cdot \mathrm{s}$ show $\mathrm{j}_{\mathrm{c}}$ from 10 to $25 \mathrm{kA} / \mathrm{cm}^{2}$ and $\mathrm{V}_{\mathrm{c}}$ up from 0.1 up to $0.5 \mathrm{mV}$ at $4.2 \mathrm{~K}$.
Good overdamped junctions have been realized also with 50 and $30 \mathrm{~nm}$ of Al. In this case even higher values of and have been measured, by using oxidation exposures from 100 to $500 \mathrm{~Pa} \cdot \mathrm{s}$. The dependence of the critical current density and the characteristic voltage on the two mentioned fabrication parameters is shown in Figs. (3 and $\mathbf{4})$.

While data show a regular dependence of $J_{c}$ on the exposure dose, a less defined dependence for this parameter on the alunminum thickness has been observed in the range from 30 to $100 \mathrm{~nm}$ (Fig. 3). On the contrary, the characteristic voltage of these junctions is strongly affected by aluminum thickness, with and evident linear relationship (see Fig. 4). The experimental data seems however to indicate

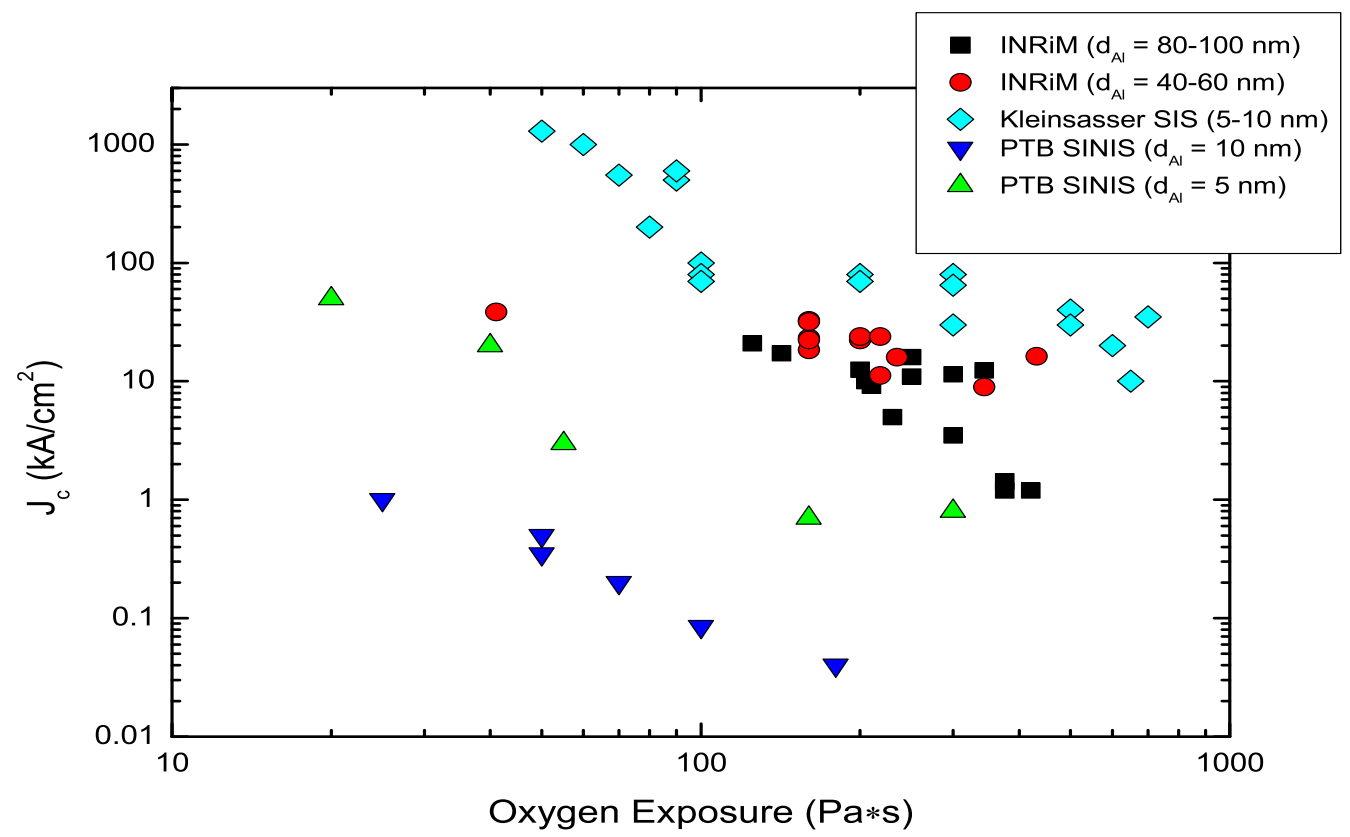

Fig. (3). Dependence of the critical current density on oxidation exposure. Square symbols indicate data corresponding to Al thickness 50 $\mathrm{nm}$, circles are data corresponding to junctions with Al thickness $>50 \mathrm{~nm}$

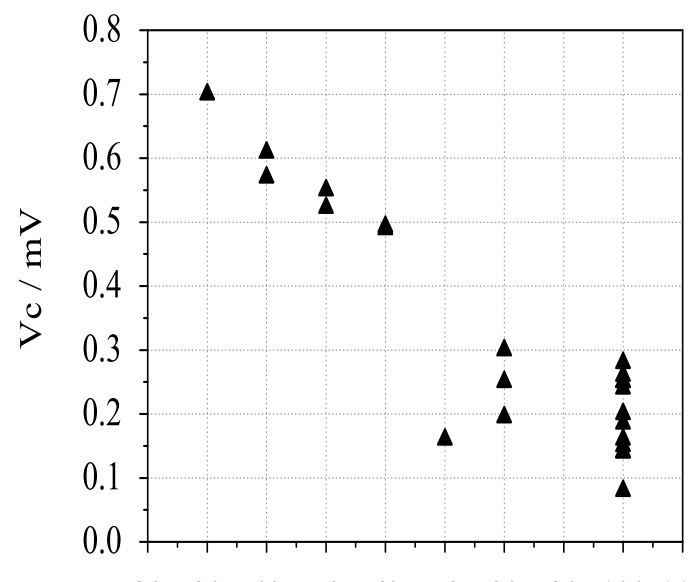

$\begin{array}{lllllllllll}20 & 30 & 40 & 50 & 60 & 70 & 80 & 90 & 100 & 110\end{array}$

Al Thickness / nm

Fig. (4). Dependence of the critical voltage on Al thickness. Circles indicate data observed on junctions with oxidation exposure < $200 \mathrm{~Pa} \cdot \mathrm{s}$, square symbols are used for data corresponding to exposures in the range 200-300 Pa.s, and triangles are results obtained with exposures 300 $\mathrm{Pa} \cdot \mathrm{s}$. 
that there is defined range of thickness of this layer where both and are maximized. A reduction of the aluminum film thickness is indeed desired, since, for instance it affects the transition temperature to the normal state of these junctions: it changes from $7.5 \mathrm{~K}$ for a $90-100 \mathrm{~nm}$ Al film up to about $8.9 \mathrm{~K}$ for a $30-40 \mathrm{~nm}$ film. Yet, anomalies of the current voltage characteristic are more easily observed for the thinner aluminum films, since high values and conditions at the border of the hysteretic to non-hysteretic transition results in more pronounced thermal instabilities of the IV characteristic.

Possible interpretations of the shunting of the thin oxide barrier must take into account several aspects. It is well known that the damping of the current voltage characteristic of a Josephson junction is defined by the value of the Stewart-McCumber parameter (see e.g. [53]): $\beta c=(2 e / \hbar)$ $I_{C} R_{N}^{2} C$.

This can be also rewritten as $\beta c=\pi \Delta r N c s / \hbar$ where $\mathrm{c}_{\mathrm{s}}$ is the junction specific capacitance, $r_{N}$ the junction normal resistivity, related to the barrier This parameter for our junctions is $<1$ at $4.2 \mathrm{~K}$, but close to unity.

In general, for different junctions configurations the product of the elementary charge $e$ and the characteristic voltage $V_{C}$ is proportional to the energy gap value of the superconducting electrodes. The usual way to decrease is to reduce $R_{N}$, that is to use ultra high transparency barriers. In [53] the replacement of $\mathrm{Nb}$ with $\mathrm{Al}$ was suggested to reduce the superconducting gap, effectively decreasing the StewartMcCumber parameter. Our junctions are not made with $\mathrm{Al}$ as an electrode material, since, as mentioned, for most of superconductive elctronics applications $\mathrm{Nb}$ is preferred, nor have such extremely thin barriers as in [53], thus the risk of pinhole defects is reduced, rather the gap value can be reduced with temperature.It should be noticed that even in the case of the presence of a pin hole, its effect is reduced by the presence of an additional normal $\mathrm{Al}$ interlayer that transforms the problem from a direct contact between the electrodes to an SNS region in parallel with the main SNIS.

Indeed our junctions, having not quite high transparency oxide barriers, look also similar in behaviour to the so called metal-to-insulator transition barrier junctions [54], and can be tuned to the hysteretic non hysteretic transition. In any case it seems that an important role is also played by the proximity effect since the thick aluminum film affect the $\mathrm{Nb} / \mathrm{Al}$ system at the interface with the oxide barrier.

\subsection{Reproducibility of Junctions Parameters, Temperature Behavior}

The reproducibility of these parameters has been tested by comparing data of 29 different devices belonging to 11 different fabrication wafers. Each wafer had an aluminum thickness of $50 \mathrm{~nm} \pm 5 \%$, while the oxidation exposure Eox was $200 \mathrm{~Pa} \cdot \mathrm{s} \pm 25 \%$. Both the wafer-to-wafer and the onwafer reproducibility have been measured. The overall average characteristic voltages measured at $4.2 \mathrm{~K}$ of both single junctions and small arrays are given in Table $\mathbf{1}$, along with the values of measured critical currents.

The principal cause of wafer to wafer reproducibility limitation arises from the spread in oxidation conditions, since the described process is presently not automated. As for the oxidation exposure, we use a dynamic process, where the introduction of oxygen flow at an initial pressure of $\mathrm{Pa}$, monitored by vacuum gauge. The on wafer reproducibility is affected by resolution of our photolitographic process (masks, resist..) and by a gradient of the deposition parameters, due to the dimensions of the cathodes and the type of sputtering process employed.

Time stability was estimated by measuring them after a chosen time lapse, from 1 to 36 month. Data for 1, 3, 12, 30 and 36 months have shown both in single junctions and arrays varying from $3 \times 10^{-3}$ and $2 \times 10^{-2}$. All these samples had undergone at least two thermal cycles. The process seems then quite insensitive to aging and cycling.

To conclude, to fabricate reproducible fully non-hysteretic junctions, the best values of $\mathrm{Al}$ thickness and oxidation exposure are in the range $40-80 \mathrm{~nm}$ and $120-350 \mathrm{~Pa} \cdot \mathrm{s}$, respectively. The restriction for $\mathrm{Al}$ to the lowest values is due to the need to have smooth interfaces, and, at the same time to keep junction transition temperature as high as possible; in fact while we measured $T_{c}$ of $8.8-9.0 \mathrm{~K}$ for al 30$50 \mathrm{~nm}, T_{c}$ was reduced to less than $8.0 \mathrm{~K}$ for Al 70-100 nm. On the contrary, a wider span of the exposure dose is preferable, as it is useful to obtain junctions with different $\mathrm{V}_{\mathrm{c}}$, and possibly some hysteresis of the $\mathrm{I}-\mathrm{V}$.

Table 1. Reproducibility Data fir SNIS Josephson Junctions. The Data Reported in the Table are Relative to Devices Fabricated with Two Different Sputtering Systems, HV rf Sputtering and UHV DC Sputtering, the Parameter Homogeneity Resulting therefore Independent of the Machine and Process. Similar Data of Reproducibility have been Obtained, on Fewer Samples, for Aluminum Thicknesses of 40 and $60 \mathrm{~nm}$

\begin{tabular}{|c|c|c|c|}
\hline & $V_{c}(\mathbf{m V})$ & $I_{c}(\mathbf{m A})$ & Comment \\
\hline \hline wafer-to wafer & $0.55 \pm 14.5 \%$ & $5.5 \pm 5-10 \%{ }^{\text {(c) }}$ & 29 devices on 11 wafers \\
\hline on-wafer & $\begin{array}{c}0.51 \pm 9.5 \% \text { wafer SNS14 } \\
0.63 \pm 4 \% \text { wafer SNIS32 }\end{array}$ & & $\begin{array}{c}\text { (b) } \\
8 \text { and } 10 \text { devices on each wafer, } \\
\text { from } 0.2 \text { to } 1 \mathrm{~cm} \text { apart. }\end{array}$ \\
\hline notes & $\begin{array}{c}\text { (a) } 6 \text { data on } 1 \text { square cm area } \\
\text { (b) } 6 \text { arrays and sub-arrays } 0.5-1 \mathrm{~cm} \text { apart }\end{array}$ & $\begin{array}{c}\text { (c) the variance is referred to the appropriate mean, } \\
\text { that is the average of the measured series arrays of } \\
100,200,400 \text { and } 1600 \text { junctions }\end{array}$ & $\begin{array}{c}\text { For all devices JJ area: } \\
5 \mu \mathrm{m} \times 5 \mu \mathrm{m}\end{array}$ \\
\hline
\end{tabular}



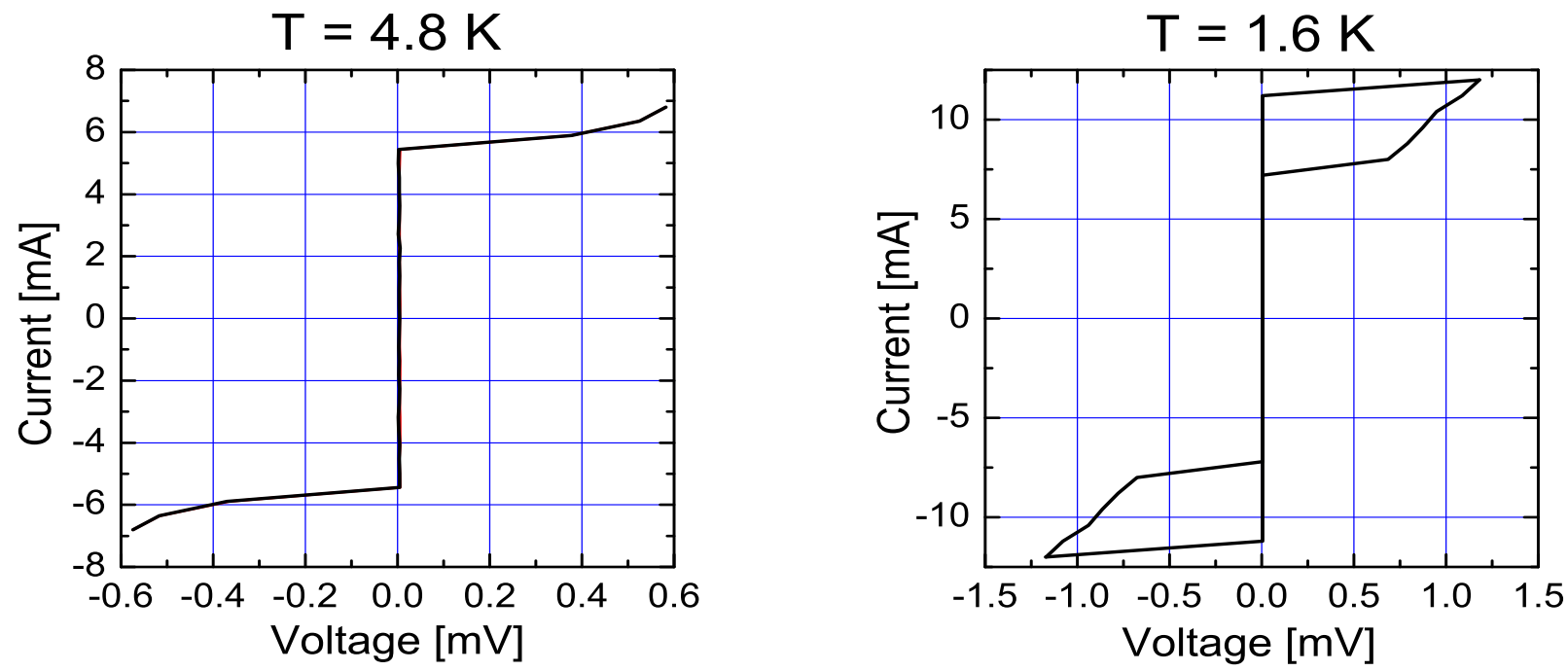

Fig. (5). IV characteristic of $\mathrm{Nb} / \mathrm{Al}-\mathrm{AlOx} / \mathrm{Nb}$ overdamped junction without microwave irradiation at different temperatures.

Measurement of the $\operatorname{Ic}(\mathrm{T})$ dependence of these junctions from $2 \mathrm{~K}$ to $9 \mathrm{~K}$, has shown different behavior as function of the aluminum film thickness. In fact for $\mathrm{Al}<50 \mathrm{~nm}$ the $\mathrm{Ic}(\mathrm{T})$ is similar to SIS Ambegaokar Baratoff dependence, while proximity effect related to the increasing of aluminum to niobium thickness, modify the Ic(T) for Al $50 \geq \mathrm{nm}$. In that case three distinct ranges are observed: 1) around $4.2 \mathrm{~K}$ the junctions behavior is dominated by the thick Al normal layer, and data are similar to those obtained for junctions with the same electrode thickness and configuration (see ref. [32]). The IV characteristic is hysteretic or non-hysteretic depending on the oxide exposure. 2) Below $4.2 \mathrm{~K}$ the junctions eventually become SS'IS, since aluminum is superconductor at $1.5 \mathrm{~K}$, and in proximity with the base niobium film undergoes a $\mathrm{N} / \mathrm{S}$ transition at a temperature intermediate between 1.5 and $4.2 \mathrm{~K}$. The current increases by a factor of 2, with respect to the value at $4.2 \mathrm{~K}$ and the junctions IV characteristic becomes hysteretic, since $\beta_{c}$ is now > 1.3) Above 4.2 K the junctions current decreases, see below, and the behaviour is SNS like, up to the transition, since $\beta_{c}$ is now $<1$. Fig. (5) show the IV characteristic of these junctions at two different temperatures. The temperature dependence of and has shown that higher values of and are obtained at temperatures below $4.2 \mathrm{~K}$, and, what is more interesting, reasonable values are still found at temperatures above $4.2 \mathrm{~K}$.

\subsection{Applications to Voltage Standard}

$\mathrm{Nb} / \mathrm{Al}-\mathrm{AlOx} / \mathrm{Nb}$, can be usefully employed in programable voltage standard since they have the following advantages with respect to other technologies.

a) High values of both current density and characteristic voltage can be obtained, compared with other types of junctions

b) Aluminum thickness and oxidation dose parameters can be selected to provide values about $150 \mu \mathrm{V}$ to optimize at $4.2 \mathrm{~K}$ for $75 \mathrm{GHz}$ irradiation.

Owing to the high values of critical current density reduction of junction size appear feasible with overdamped
$\mathrm{Nb} / \mathrm{al}-\mathrm{AlOx} / \mathrm{Nb}$. A reduction of the array area of a factor 4 at least is predictable. This will help homogeneity of the fabrication process and of the rf power distribution.

A further possibility would be to use junctions with $V_{c}$ as high as $500 \mu \mathrm{V}$ and therefore radiating the junctions with microwave signals at frequencies > $100 \mathrm{GHz}$; this could reduce the number of junctions needed to obtain $10 \mathrm{~V}$ output.

$\mathrm{Nb} / \mathrm{Al}-\mathrm{AlOx} / \mathrm{Nb}$ overdamped junctions parameters can be set to fabricate junctions showing no hysteresis, with a purely SNS-like behavior, thus are suitable for PJAVS.

RSFQ circuits to drive the Josephson array can be realized with SNIS technology, in this case realizing trilayers with dedicated parameters, i.e. with $V_{c}$ as high as possible, > $0.5 \mathrm{mV}$ at $4.2 \mathrm{~K}$.

\section{OPERATION OF VOLTAGE REFERENCES ABOVE 4.2 K}

An interesting issue is in the possibility of operation at temperatures higher than $4.2 \mathrm{~K}$, in view of the substitution of expensive liquid helium refrigeration systems with the compact cryocoolers which can lead the diffusion of voltage standards to the private companies.

This is a challenge of great importance for superconductive circuits and voltage standards apparently not to be solved in a short period by high $T_{c}$ junctions. At present, large arrays fabricated with higher critical temperature superconductors, like YBCO or the more recent $\mathrm{MgB} 2$, are not yet available, since the technology of these junctions do not allow to achieve the required integration level. One big problem of these junctions is the stability in time and with thermal cycling.

The most interesting results sofar have been achieved with YBCO bicrystal shunted junctions, where quantized steps have been measured near $77 \mathrm{~K}$ at $28 \mathrm{mV}$.

Concerning niobium and niobium nitride based junctions, only $\mathrm{NbN} / \mathrm{TiN} / \mathrm{NbN}$ [35] have demonstrated operation at 
these temperatures, achieving a sound result such as a 11 bit DAC with 10 and also $20 \mathrm{~V}$ output at $10 \mathrm{~K}$.

However, they are sensitive to temperature changes, requiring a stabilization of the cryocooler at $0.1 \mathrm{~K}$ level, have a strong demand on dissipated power and require a toplevel, costly, fabrication process.

As for niobium based junctions, SINIS junctions even if have different temperature dependence, depending on the multilayer configuration, the most reproducible devices with symmetric barriers have shown values of Ic generally too low at $\mathrm{T}>4.2 \mathrm{~K}[29,33]$.

Among SNS, all the pure metallic barrier are not suitable for operation at $\mathrm{T}$ above liquid helium, since their $V_{c}$ is too low. Only MoSi barrier have a different temperature dependence depending on barrier thickness and for instance, show a reduction of of 0.2 at $4.2 \mathrm{~K}$ at $7.5 \mathrm{~K}$, which gives a best $V_{c}$ of $30 \mu \mathrm{V}$ [43].

Different from these, the NbSi barrier junctions could provide higher values of Ic and $V_{c}$ at $\mathrm{T}>4.2 \mathrm{~K}$, (see ref. [42]). For these junctions the temperature dependence should still be carefully examined, since in this case the almost semiconductive barrier can have a temperature dependent stability.

In this context, $\mathrm{Nb} / \mathrm{Al}-\mathrm{AlOx} / \mathrm{Nb}$ overdamped junctions realized at INRiM are also appealing, since they can be operated at temperatures close to $\mathrm{Nb}$ transition, between 8 and $9 \mathrm{~K}$.

First measurement of the quantized voltage steps, radiating the junctions with a $75 \mathrm{GHz}$ source, have shown that an optimal working condition can be obtained at such high temperatures, due to the fact that the amplitude of the $n=1$ step normalized to the critical current attains its maximum when the microwave drive frequency fd and the junction characteristic frequency fc are nearly equal. $\mathrm{fd}=\mathrm{fc}$ corresponds also to a condition of minimal power dissipation [22]. The reduction of at temperatures as high as $7 \mathrm{~K}$ is then not critical, since it is partly compensated by the optimal fc/fd ratio. In particular, we have measured on a single junction an amplitude of the $\mathrm{n}=1$ step of about $1 \mathrm{~mA}$ at $\mathrm{T}=8.3 \mathrm{~K}$ on a 5 $\mu \mathrm{m}$ size single junction, flat at $\mathrm{nV}$ level [52]. This result has been confirmed by preliminary measurements on small series arrays of 10 and 100 junctions, where a step of hundreds of $\mu \mathrm{A}$ has been observed up $7 \mathrm{~K}$, at voltages of 1.4 and $14 \mathrm{mV}$ respectively (See Fig. 6).

This possibility should of course more deeply investigated, by measuring the step amplitude at various temperatures for increasing number of junctions, in order to establish if any reduction of operative margins is produced [51].

The reduction of the working temperature increasing the number of junctions is presently due to a poor thermal exchange between the array and the corning glass substrate 1 $\mathrm{mm}$ thick, along with the non-optimal coupling between $\mathrm{rf}$ signal and the array. Improved results are expected by using thinner silicon substrate and a refined design of microstripline structures.

\section{CONCLUSIONS}

The extension of DC Josephson Voltage standard to AC has led to the development of different type of circuits, according to the specific application.

In section 2 a comparison of different types of overdamped junctions developed for the large circuits needed by the AC extension of Josephson voltage standard has shown advantages and disadvantages of a certain electrode configuation or material. In order to achieve the desired operating conditions, $V_{c}$ must be fitted to the drive frequency (i.e. 100 $150 \mu \mathrm{V}$ for $70 \mathrm{GHz}$ ). The requirements for $J_{c}$ are different: for low $J_{c}$ the size of the JJ is large, for high $J_{c}$ (above 100 $\mathrm{kA} / \mathrm{cm} 2$ ) the technological efforts increase significantly (sub- $\mu \mathrm{m} \mathrm{JJ}$, connection of the wiring to sub- $\mu \mathrm{m}$ electrodes with high-current capability, improved cooling needed due to self-heating of $\mathrm{JJ}$ in highly-integrated series arrays). And these issues must be attained using a tolerable degree of technological effort, also in terms of allowable integration. In fact, in order to have a widespread development in the world laboratories for these circuits, neither the number of junctions should be increased nor the size reduced beyond a certain limit. All together, these issues are not solved by any

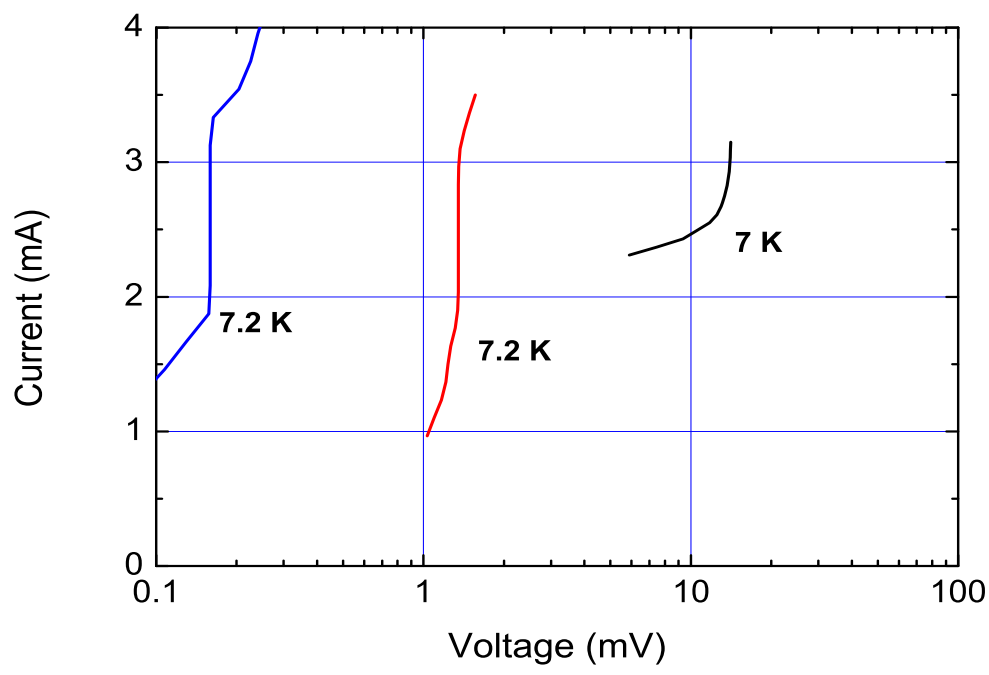

Fig. (6). Quantized steps induced at different voltage levels by a $75 \mathrm{GHz}$ microwave signal are presented at different temperatures. A step 200-300 $\mu \mathrm{A}$ wide is still observed at 7.2 K, while amplitudes around $1 \mu \mathrm{A}$ are measured at lower temperatures. 
of the junctions reported in this analysis, even if some of the described technologies had achieved sound results.

As for SINIS junctions, which has been the most established and successful process in past years, and has been at the basis of both binary array standard at $1 \mathrm{~V}$ and of PWM and RSFQ circuits, while it allows to use these junctions with microwave drive frequencies of about 75 $\mathrm{GHz}$, thereby reducing their number, it does not allow to obtain adequate values of $J_{c}$, therefore requiring a large size. Therefore, no really compact integration of devices is possible while in any case their fabrication process is not trivial, making it difficult to fabricate arrays for voltage up to $10 \mathrm{~V}$ and more.

Among SNS junctions, which typically have adequate $J_{c}$ values, the critical issue is a $V_{c}$ value sufficiently high. Therefore a huge number of junctions is necessary to achieve quantized voltages with adequate span., even if they are well suited to pulse driven voltage standards.

For this type of standards three possibilities have been mainly addressed:

- submicron junctions such as $\mathrm{Nb} / \mathrm{HfTi} / \mathrm{Nb}$;

- extremely top-level technology as NbN/TiN/NbN;

- multistacked lumped arrays as $\mathrm{Nb} / \mathrm{MoSix} / \mathrm{Nb}$ and $\mathrm{Nb} /$ $\mathrm{NbxSix/Nb}$

While each of these three technologies has indeed achieved important results, and it is a good candidate for AC synthesis with enough voltage resolution, all of them require hundreds of thousands of junctions to obtain voltages higher than $1 \mathrm{~V}$ and none of them is in any case a good solution for other superconductive electronics circuits, since none of them feature a $V_{c}$ higher than $200 \mu \mathrm{V}$.

Very promising results have been recently reported on $\mathrm{NbSi}$ barrier junctions, where by trimming the stoichiometry and the thickness of the barrier $V_{c}$ and $J_{c}$ have been varied in a wide range, and the homogeneity of the process has allowed the fabrication of large circuits.

In section 3 results on $\mathrm{Nb} / \mathrm{Al}-\mathrm{AlOx} / \mathrm{Nb}$ overdamped SNIS junctions developed at INRiM show the advantages of a really simple technology, which neither requires deep submicron dimensions nor implies the use of extremely large number of junctions, featuring high values of both $J_{c}$ and $V_{c}$. The possibility of realizing junctions with some hysteresis, results also useful for a type of AC standard where pulse modulation is performed by the so-called PWM scheme.

A last major issue of next generation of Voltage standards discussed briefly in section 4 is the possibility of operation above $4.2 \mathrm{~K}$, with considerable advantage for power dissipation.

For this, apart high $T_{c}$ junctions, still to be improved in reproducibility, only very complex $\mathrm{NbN} / \mathrm{TiN} / \mathrm{NbN}$ juctions have proved to work at temperatures as high as $10 \mathrm{~K}$. The possibility of achieving quantized step of sufficient amplitude $(1 \mathrm{~mA})$ also at temperatures as high as $7-8 \mathrm{~K}$ in single junctions and something less in 10 and 100 junctions series arrays, on the other hand paves the way to experimental setup with a simpler refrigeration support also for SNIS junctions based on niobium.

\section{REFERENCES}

[1] Levinsen MT, Chiao RY, Feldman MJ, et al. An inverse ac Josephson effect voltage standard. Appl Phys Lett 1977; 31(11): 776-8.

[2] Hamilton CA, Burroughs CJ, Kautz RL. Josephson d/a converter with fundamental accuracy. IEEE Trans Instrum Meas 1995; 44(2): 223-5.

[3] Behr R, Kohlmann J, Janssen JTBM, et al. Analysis of different measurement setups for a programmable Josephson voltage standard. IEEE Trans Instrum Meas 2003; 52(2): 524-8.

[4] Geneves G, Gournay P, Gosset A, et al. The BNM watt balance project. IEEE Trans Instrum Meas 2005; 54(2): 850-3.

[5] Burroughs CJ, Benz CP, Dresselhaus PD, et al. Development of a $60 \mathrm{~Hz}$ Power Standard Using SNS Programmable Josephson Voltage Standards. IEEE Trans Instrum Meas 2007; 56(2): 289-94.

[6] Yamamori Y, Yamada T, Sasaki H, et al. A $10 \mathrm{~V}$ programmable Josephson voltage standard circuit with a maximum output voltage of 20 V. Supercond Sci Technol 2008; 21(10): 105007.

[7] Kohlmann J, Behr R, Funck T. Josephson voltage standards. Meas Sci Technol 2003; 14(8): 1216-28.

[8] Monaco R. Enhanced AC Josephson effect. J Appl Phys 1990; 68: 679-87.

[9] KimJ-H, Sosso A. AF Clark dynamics of overdamped Josephson junctions driven by a square-wave pulse. J Appl Phys 1998; 83(6): 3225-32.

[10] Sosso A. Phase lock of non-hysteretic Josephson junctions with pulse bias: analytical properties. Philos Mag Part B 2000; 80(5): 973-7.

[11] Benz SP, Hamilton CA. A pulse-driven programmable Josephson voltage standard. Appl Phys Lett 1996; 68: 3171-3.

[12] Benz SP, Hamilton CA, Burroughs CJ, et al. AC and DC bipolar voltage standard using quantized pulses. IEEE Trans Instrum Meas 1999; 48: 266-9

[13] Kohlmann J, Kieler OF, Iuzzolino R, et al. Development and investigation of SNS Josephson arrays for the Josephson arbitrary waveform synthesizer. IEEE Trans Instrum Meas 2009; 58(4): 797 802 .

[14] Katkov AS, Klushin AM, Telitchenko GP, et al. Challenges of Josephson junction arrays for AC voltage generation by microwave pulse power modulation. IEEE Trans Appl Supercond 2005; $15(2$ Pt 1): 352-5.

[15] Ter Brake HJM, Buchholz FIM, Burnell G, et al. SCENET roadmap for superconductor digital electronics. Physica C 2006; 439(1): 1-41.

[16] Semenov VK. Digital to analog conversion based on processing of the SFQ pulses. IEEE Trans Appl Supercond 1993; 3(1 Pt 4): 2637 40 .

[17] Likharev KK, Semenov VK. RSFQ logic/memory family: a new Josephson-junction technology for sub-Teraherz-clock-frequency digital systems. IEEE Trans Appl Supercond 1991; 1(1): 3-28.

[18] Semenov VK, Voronova MA. DC voltage multipliers - A novel application of synchronization in Josephson junction arrays. IEEE Trans Magn 1989; 25: 1432-5

[19] Hamilton CA. Josephson voltage standard based on single-fluxquantum voltage multipliers. IEEE Trans Appl Supercond 1992; 2(3): 139-42.

[20] Maezawa M, Hirayama F. 10-bit rapid single flux quantum digitalto-analog converter for AC voltage standard. Institute of Physics Publishing. J Phys (Conf. Ser.) 2008; 97: 012161.

[21] Niemeyer J. Josephson arrays for DC and AC metrology. Supercond Sci Technol 2000; 13(5): 546-50.

[22] Kautz RL. Shapiro steps in large-area metallic-barrier Josephson junctions. J Appl Phys 1995; 78: 5811-8.

[23] Klushin AM, He M, Yan SL, et al. Arrays of high-Tc Josephson junctions in open millimeter wave resonators. Appl Phys Lett 2006; 89: 232505(1-3)

[24] Cybart SA, Chen K, Cui Y, et al. Planar MgB2 josephson junctions and series arrays via nanolithography and ion damage. Appl Phys Lett 2006; 88: 012509.

[25] Patel V, Lukens JE. Self-shunted Nb/AlO x/Nb Josephson junctions. IEEE Trans Appl Supercond 1999; 9(2): 3247-50.

[26] Golubov AA, Yu, Il'ichev E. The current-phase relation in Josephson junctions. Rev Mod Phys 2004; 76: 411-69.

[27] Capogna L, Blamire MG. Superconducting proximity effect through high-quality high-conductance tunnel barriers. Phys Rev B $1996 ; 53(9)$ : 5683-7. 
[28] Mueller F, Behr R, Palafox L, et al. Improved 10 V SINIS Series Arrays for Applications in AC Voltage Metrology. IEEE Trans Appl Supercond 2007; 17(2): 649-52.

[29] Kohlmann J, Muller F, Behr R, et al. SINIS junction series arrays for the Josephson arbitrary waveform synthesizer. IEEE Trans Appl Supercond 2005; 15(2 Pt 1): 121-4.

[30] Behr R, Williams JM, Patel F, et al. Synthesis of precision AC waveform using a sinis Josephson array. IEEE Trans Instrum Meas 2005; 54: 612-5.

[31] Kohlmann J, Mueller F, Kieler O, et al. Josephson series arrays for programmable 10-V SINIS josephson voltage standards and for josephson arbitrary waveform synthesizers based on SNS junctions. IEEE Trans Instrum Meas 2007; 56(2): 472-5.

[32] Nevirkovets IP, Shafranjuk SE, Ketterson JB, et al. Properties of high-J $\mathrm{J}_{\mathrm{c}}$ SINIS junctions. IEEE Trans Appl Supercond 2003; 13(2): 1085-8.

[33] Born F, Cassel D, Ilin K, et al. Transport properties of SINIS junctions with high-current density. IEEE Trans Appl Supercond 2003; 13(2): 1079-84.

[34] Mueller F, Behr R, Weimann T, et al. $1 \mathrm{~V}$ and $10 \mathrm{~V}$ SNS programmable voltage standards for $70 \mathrm{GHz}$. IEEE Trans Appl Supercond 2009; 975-80.

[35] Yamamori H, Ishizaki M, Shoji A, et al. $10 \mathrm{~V}$ programmable Josephson voltage standard circuits using $\mathrm{NbN} / \mathrm{TiN} / \mathrm{NbN} / \mathrm{TiN} / \mathrm{NbN}$ double-junction stacks. Appl Phys Lett 2006; 88(4): 042503(1-3).

[36] Yamamori H, Ishizaki M, Sasaki H, et al. Operating margins of a $10 \mathrm{~V}$ programmable josephson voltage standard circuit using $\mathrm{NbN} / \mathrm{TiN} / \mathrm{NbN} / \mathrm{TiN} / \mathrm{NbN}$ double-junction stacks. IEEE Trans Appl Supercond 2007; 17(2 Part 1): 858-63.

[37] Freericks JK, Nikolic BK, Miller P. Tuning a Josephson junction through a quantum critical point. Phys Rev B 2001; 64(5): 054511(1-13).

[38] Freericks JK, Nikolic BK, Miller P. Superconductor-correlated metal-superconductor Josephson junctions: an optimized class for high speed digital electronics. IEEE Trans Appl Supercond 2003; 13(2): 1089-92.

[39] Lacquaniti V, Gonzini S, Maggi S, et al. Nb-based SNS junctions with $\mathrm{Al}$ and $\mathrm{TaO}$ barriers for a programmable Josephson voltage standard. IEEE Trans Appl Supercond 1999; 9(2): 4245-8.

[40] Kaul AB, Whiteley SR, Van Duzer T, et al. Internally shunted sputterd nbn Josephson junctions with a Tax barrier for nonlatching logic applications. Appl Phys Lett 2001; 78(1): 99-101.

[41] Barrera AS, Beasley BR. High-resistance SNS sandwich-type Josephson junctions. IEEE Trans Magn 1987; MAG-23(2): 866-8.
[42] Baek B, Dresselhaus PD, Benz SP. Co-sputtered amorphous Nb/Si barriers for Josephson-junction circuits. IEEE Trans Appl Supercond 2006; 16(4): 1966-70.

[43] Chong Y, Dresselhaus PD, Benz SP. Electrical properties of $\mathrm{Nb} / \mathrm{MoSi} / \mathrm{Nb}$ Josephson junctions. Appl Phys Lett 2005; 86: 232505.

[44] Chong Y, Dresselhaus PD, Benz SP, et al. Effects of Interlayer electrode thickness in $\mathrm{Nb} /(\mathrm{MoSi} / \mathrm{Nb})$ stacked Josephson junctions. Appl Phys Lett 2003; 82: 2467-9.

[45] Katkov AS, Klushin AM, Telitchenko GP, et al. Challenges of Josephson junction arrays for ac voltage generation by microwave pulse power modulation, IEEE Trans Appl Supercond 2005; 15(2): $352-5$.

[46] Karpov OV, Buchstaber VM, Sherstobitov SV, et al. Josephson junction parameters for alternating current wave form synthesis. J Appl Phys 2006; 100: 093907(1-6).

[47] Process RSFQ1D-1.2 Rapid Single Flux Quantum (RSFQ) - design rules for Nb/A12O3-A1/Nb-process, IPHT RSFQ, Available from: http://www.ipht-jena.de

[48] Maezawa M, Hirayama V. 10-bit rapid single flux quantum digitalto-analog converter for AC voltage standard. J Phys (Conf. Ser.) 2008; 97: 012161(1-5).

[49] Balashov D, Khabipov M, Buchholz F-I, et al. SINIS process development for integrated circuits with characteristic voltages exceeding $250 \mu \mathrm{V}$. IEEE Trans Appl Superconduct 2001; 11(1): 1070-3.

[50] Olaya D, Baek B, Dresselhaus PD, et al. High-Speed Nb/Nb-Si/Nb Josephson junctions for superconductive digital electronics. IEEE Trans Appl Supercond 2008; 18(4): 1797-800.

[51] Lacquaniti V, Cagliero C, Maggi S, et al. Overdamped Nb/Al$\mathrm{AlO} / \mathrm{Nb}$ Josephson junctions. Appl Phys Lett 2005; 86(4): 042501(1-3).

[52] Lacquaniti V, Andreone D, De Leo N, et al. Analysis of the Temperature Stability of Overdamped Nb/Al-AlO/Nb Josephson Junctions. IEEE Trans Appl Supercond 17(2): 609-12.

[53] Lotkhov SV, Tolkacheva EM, Balashov DV, et al. Low hysteretic behavior of Al/AlOx/Al Josephson junctions. Appl Phys Lett 89: 132115(1-3).

[54] Yu L, Gandikota R, Singh RK, et al. Internally shunted josephson junctions with barriers tuned near the metal-insulator transition for RSFQ logic applications. Supercond Sci Technol 2006; 19(8): 71931.

This is an open access article licensed under the terms of the Creative Commons Attribution Non-Commercial License (http://creativecommons.org/ licenses/by-nc/3.0/), which permits unrestricted, non-commercial use, distribution and reproduction in any medium, provided the work is properly cited. 